\title{
Estimating survival time of patients with glioblastoma multiforme and characterization of the identified microRNA signatures
}

\author{
Srinivasulu Yerukala Sathipati ${ }^{1}$, Hui-Ling Huang ${ }^{1,2}$ and Shinn-Ying $\mathrm{Ho}^{1,2^{*}}$ \\ From 15th International Conference On Bioinformatics (INCOB 2016) \\ Queenstown, Singapore. 21-23 September 2016
}

\begin{abstract}
Background: Though glioblastoma multiforme (GBM) is the most frequently occurring brain malignancy in adults, clinical treatment still faces challenges due to poor prognoses and tumor relapses. Recently, microRNAs (miRNAs) have been extensively used with the aim of developing accurate molecular therapies, because of their emerging role in the regulation of cancer-related genes. This work aims to identify the miRNA signatures related to survival of GBM patients for developing molecular therapies.

Results: This work proposes a support vector regression (SVR)-based estimator, called SVR-GBM, to estimate the survival time in patients with GBM using their miRNA expression profiles. SVR-GBM identified 24 out of 470 miRNAs that were significantly associated with survival of GBM patients. SVR-GBM had a mean absolute error of 0.63 years and a correlation coefficient of 0.76 between the real and predicted survival time. The 10 top-ranked miRNAs according to prediction contribution are as follows: hsa-miR-222, hsa-miR-345, hsa-miR-587, hsa-miR-526a, hsa-miR-335, hsa-miR-122, hsa-miR-24, hsa-miR-433, hsa-miR-574 and hsa-miR-320. Biological analysis using the Kyoto Encyclopedia of Genes and Genomes (KEGG) pathway on the identified miRNAs revealed their influence in GBM cancer.

Conclusion: The proposed SVR-GBM using an optimal feature selection algorithm and an optimized SVR to identify the 24 miRNA signatures associated with survival of GBM patients. These miRNA signatures are helpful to uncover the individual role of miRNAs in GBM prognosis and develop miRNA-based therapies.
\end{abstract}

\section{Background}

Glioblastoma multiforme (GBM) is the most common malignant human brain tumor [1]. There are two subtypes of glioblastoma, primary glioblastoma and secondary glioblastoma, which originate from different genetic pathways and affect patients of different ages [2]. Generally, standard therapies, such as radiotherapy and chemotherapy, do not contribute better survival benefits to GBM patients due to tumor reoccurrences even after

\footnotetext{
* Correspondence: syho@mail.nctu.edu.tw

${ }^{1}$ Institute of Bioinformatics and Systems Biology, National Chiao Tung University, Hsinchu, Taiwan

2Department of Biological Science and Technology, National Chiao Tung University, Hsinchu, Taiwan

multimodality treatment [3]. GBM patients' median survival rate is very poor ranging from 12 to 14 months [4]. Early stage detection approaches are necessary to better understand the events in GBM and for the development of therapeutics.

MiRNA is a small ( 18-22 nucleotides) non-coding RNA which targets messenger RNA (mRNA) for translation inhibition, thereby regulating protein expression [5]. MiRNA regulates several biological processes, such as cell proliferation [6], haematopoiesis [7], insulin secretion and apoptosis $[8,9]$. Nowadays, miRNA expression profiling is extensively used in cancer studies due to its effective role in identifying cancer gene expression regulations. Many profiling studies have reported altered 
miRNA expressions in different cancers, including lung cancer, colon cancer, leukaemia, and glioblastoma [10-13]. Over the last several years, molecular characteristics have been used to predict tumor grades as well as to identify the microarrays which are associated with patient survival [14-16]. The combination of gene expression profiles and machine learning approaches have often been used to predict risk assessment, cancer recurrence and survivability, and to identify the potential biomarkers associated with cancer treatment. Gene expression profiling was used to identify genes which can classify different grades of tumors in GBM patients [17]. Fuller et al. used the microarray technology and k-nearest neighbour algorithm to classify tumor types in glioma patients [18]. Moreover, it was proven that miRNA expression profiles are more accurate in classifying different tumor types when compared with mRNA expression profiles [19]. Several studies reported that miRNA expression alterations have prognostic significance and are associated with overall survival among patients with GBM [20-22]. Recent miRNA-based studies revealed that miRNA expression is associated with chemo-resistance and radio-resistance [23, 24]. In conclusion, cancer treatment therapy based on miRNA expression profiles better contributes to the development of novel treatment and diagnosis approaches in patients with GBM.

Teplyuk et al. obtained promising accuracy using miRNA profiling of cerebrospinal fluid to develop a support vector machine model which distinguishes the glioblastoma and metastatic brain tumors [25]. Roth et al. distinguish glioblastoma patients from healthy controls using a support vector machine in order to identify the tumor-specific miRNAs and achieved an accuracy, sensitivity and specificity of 81,83 , and $79 \%$, respectively [26]. A k-nearest neighbour method has been used to classify high-grade gliomas based on gene expression profiles and it was observed that the prediction models led to better clinical outcomes by separating diagnostically challenging malignant gliomas [27]. Current studies of prediction methods have used small datasets and the majority of proposed methods are concerned with detection and classification of different types of tumors and malignancies.

However, before miRNA expression profiling can be implemented in clinical practice, effective methods which can be applied to large datasets are still needed for the development of potential therapeutics associated with patients' survival. Accordingly, this work proposes a support vector regression (SVR)-based method, called SVR-GBM, for identification of miRNAs to estimate the survival time in patients with GBM. High performance of SVR-GBM was derived from an optimal feature selection method, inheritable bi-objective combinatorial genetic algorithm (IBCGA) [28]. In this work, we utilized the cancer genome atlas (TCGA) data portal to obtain miRNA expression profiles of 247 patients with GBM. SVR-GBM identified 24 out of 470 miRNAs for the prediction of survival time in patients with GBM and obtained a mean absolute error of 0.63 years and a correlation coefficient of 0.76 between the real and predicted survival time. Further, we ranked these miRNAs based on their contribution to the survival time prediction. The biological significance of the 10 top-ranked miRNAs in cancer pathways was analysed. The identified miRNA signatures may help to develop miRNA-based therapies in GBM medicine.

\section{Results and Discussion}

\section{Estimation of survival time}

We made an attempt to estimate survival time of GBM cancer patients using their miRNA expression profiles. We utilized 247 patients with GBM and the survival time of these patients was between 0.4 to 11 years. SVR-GBM used an optimal feature selection algorithm IBCGA to identify 24 out of 470 miRNAs which are associated with survival time of cancer patients. This study is the first to use a support vector regression model combining with an optimal feature selection of miRNAs to estimate survival time among patients with GBM. SVR-GBM achieved a correlation coefficient of 0.76 and a mean absolute error of 0.63 years using 10 -fold cross-validation. The correlation plot between real and predicted survival time is shown in Fig. 1.

We employed multiple regression analysis using the stepwise feature addition method [29] and elastic net [30] to compare with SVR-GBM. The comparison results are shown in Table 1. SVR-GBM achieved a correlation coefficient, mean absolute error, and standard error of estimates of $0.76,0.63$ years and 11.34 , respectively; better than the multiple linear regression with the correlation coefficient, mean absolute error, and standard error of estimates of $0.63,0.80$ years and 13.97, respectively; and the elastic net method with the correlation coefficient, mean absolute error, and standard error of estimates of $0.39,0.86$ years and 16.35 , respectively.

\section{Ranks of the identified miRNA signatures}

We performed a main effect difference (MED) analysis to reveal the contribution of each miRNA to the survival prediction model by an orthogonal experimental design [31]. The 24 identified miRNAs and MED scores are shown in Table 2. The 10 top-ranked miRNAs using the MED analysis are hsa-miR-222, hsa-miR-345, hsa-miR-587, hsa-miR-526a, hsa-miR-335, hsa-miR-122, hsa-miR-24, hsa-miR-433, hsa-miR-574, and hsa-miR-320. Furthermore, we assessed the biological significance of these 10 miRNAs using the KEGG pathway analysis. 


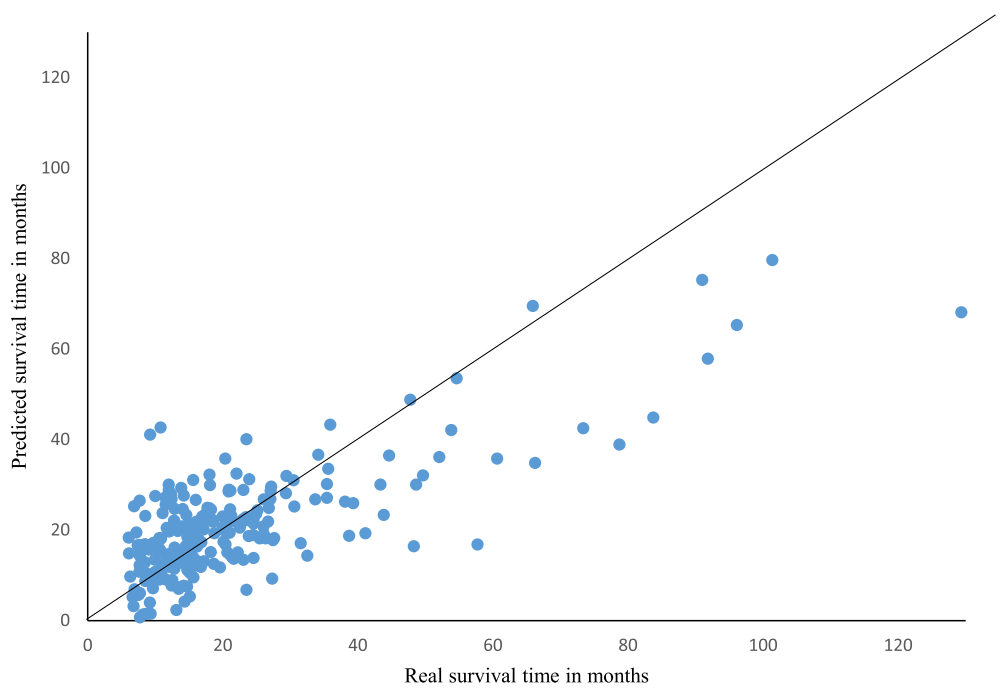

Fig. 1 Predicted survival time on $\mathrm{Y}$-axis and real survival time on $\mathrm{X}$-axis

\section{Characteristics of the identified miRNAs}

1) Hsa-miR-222: This miRNA plays a critical role in GBM intervention. Hsa-miR-221/222 are often upregulated in GBM. This miRNA regulates cell proliferation in U251 glioma cells by targeting the functional p27kip1 gene (a member of the kip family of cyclin-dependent kinase inhibitors) [32], and co-suppression of this miRNA by the antisense approach inhibits advanced tumor cell proliferation and may function as a potential therapeutic in glioma [32]. Zhang et al. found the inverse relation between mir-222 and pro-apoptotic genes in glioma cells [33]. Alteration of this miRNA in glioma cells upregulates PUMA expression and promotes apoptosis, thus reducing tumor size [33]. In addition, investigation of glioma cell lines revealed that hsa-miR-222 also targets the gene TIMP2, suppression of this miRNA regulated cell invasion and angiogenesis [34]. Experimental validation in malignant glioma cells concluded that mir-222 acts as an oncogenic by targeting connexin $43(\mathrm{Cx} 43)$ and regulating cell proliferation and invasion [35]. Moreover, mir-222 plays an important role in small cell lung cancer and hepatocellular carcinoma by targeting phosphate and tensin homolog and the tissue inhibitors of metallo-proteinase tumor suppressors and by enhancing cellular migration [36].
2) Hsa-miR-345: Zinn et al. reported that hsa-miR-345 was correlated with short survival times in glioblastoma patients [37]. We observed that though the participation of hsa-miR-345 is limited in glioblastoma, it's expression is often deregulated in other major cancer types. For instance, hsa-miR-345 has been found to be deregulated in non-small cell lung cancer, and its expression is associated with clinicopathelogical features [38]. In prostate cancer, mir-345 regulates cell proliferation, invasion, and migration by targeting the Smad1 gene [39]. Luciferase assay analysis reported that BCL-2 associated anthanogene- 3 is the target of mir-345, and over expression of this miRNA suppresses cell proliferation and invasion in colorectal cancer cells in vitro [40].

3) Hsa-miR-335: A real-time quantitative RT-PCR assay study reported that the expression of hsa-miR-335 is significantly associated with the clinicopathelogical factors and survival time of patients with GBM. It was also noted that expression levels of mir-335 were higher in a short survival group, when compared with a long survival group [41]. In most cases, it was downregulated in breast and ovarian cancers. In breast cancer cell lines, mir-335 targets three prime untranslated regions of c-Met and subsequently inhibits cell migration [42]. Mir-335 expression is down-regulated in ovarian cancer cell lines when compared with adjacent normal counterparts [43]. In neuroblastoma,

Table 1 Prediction performance of SVR-GBM

\begin{tabular}{llccr}
\hline Method & Features selected & Correlation coefficient & Mean absolute error (MAE) & Standard error of estimates \\
\hline SVR-GBM & 24 & 0.76 & 0.63 & 11.34 \\
Multiple regression analysis & 15 & 0.63 & 0.80 & 13.97 \\
Elastic net & 6 & 0.39 & 0.86 & 16.35 \\
\hline
\end{tabular}


Table 2 Results of the main effect difference analysis. 24 miRNA sequences and corresponding MED scores

\begin{tabular}{lll}
\hline miRNA & MED & Mature sequence \\
\hline hsa-miR-222 & 0.796768 & AGCUACAUCUGGCUACUGGGU \\
hsa-miR-345 & 0.567302 & GCUGACUCCUAGUCCAGGGCUC \\
hsa-miR-587 & 0.535874 & UUUCCAUAGGUGAUGAGUCAC \\
hsa-miR-526a & 0.461675 & CUCUAGAGGGAAGCACUUUCUG \\
hsa-miR-335 & 0.457645 & UCAAGAGCAAUAACGAAAAUGU \\
hsa-miR-122 & 0.443237 & UGGAGUGUGACAAUGGUGUUUG \\
hsa-miR-24 & 0.427016 & UGGCUCAGUUCAGCAGGAACAG \\
hsa-miR-433 & 0.40424 & AUCAUGAUGGGCUCCUCGGUGU \\
hsa-miR-574 & 0.338432 & CACGCUCAUGCACACACCCACA \\
hsa-miR-320 & 0.337497 & AAAAGCUGGGUUGAGAGGGCGA \\
hsa-miR-768 & 0.304775 & GUUGGAGGAUGAAAGUACGGAGUGAU \\
hsa-miR-223 & 0.287394 & CGUGUAUUUGACAAGCUGAGUU \\
hsa-miR-497 & 0.266012 & CAGCAGCACACUGUGGUUGU \\
hsa-miR-370 & 0.220475 & CAGGUCACGUCUCUGCAGUUAC \\
hsa-miR-137 & 0.219401 & UUAUUGCUUAAGAAUACGCGUAG \\
hsa-miR-605 & 0.210376 & UAAAUCCCAUGGUGCCUUCUCCU \\
hsa-miR-491 & 0.207076 & AGUGGGGAACCCUUCCAUGAGG \\
hsa-miR-656 & 0.204699 & AGGUUGCCUGUGAGGUGUUCA \\
hsa-miR-15b & 0.170935 & UAGCAGCACAUCAUGGUUUACA \\
hsa-miR-801 & 0.170311 & GAUUGCUCUGCGUGCGGAAUCGAC \\
hsa-miR-221 & 0.129155 & ACCUGGCAUACAAUGUAGAUUU \\
hsa-miR-95 & 0.104175 & UCAAUAAAUGUCUGUUGAUUU \\
hsa-miR-603 & 0.099838 & CACACACUGCAAUUACUUUUGC \\
hsa-miR-519C & 0.02755 & CUCUAGAGGGAAGCGCUUUCUG \\
\hline & & \\
\hline
\end{tabular}

mir-335 regulates the transforming growth factor- $\beta$ (TGF- $\beta$ ) non-canonical pathway and inhibits the transient potential of neuroblastoma cells [44].

4) Hsa-miR-24: A qRT-PCR assay study reported that hsa-miR-24 acts as an oncogene that directly targets ST7L and suppresses the $\beta$-catenin/ Tcf 4 transcription activity, and that further suppression of this miRNA expression regulates cell proliferation and invasion in glioma cells [45]. MTT assay analysis revealed that hsa-miR-24 targets the MXI1 tumor suppressor gene and promotes cell proliferation, and that it is upregulated in glioma cells [46]. Upregulation of mir-24 was also observed in breast and nonsmall cell lung cancers. In breast cancer, mir-24 directly targets the p27Kip1 and inhibits apoptosis in MDA-MB-435 and MDA-MD-468 cells [47], as well as in non-small cell lung cancer cells. This miRNA targets nuclear apoptosis-inducing factor 1 and induces cell proliferation [48].

5) Hsa-miR-320: Quantitative real-time PCR analysis was used to assess human glioma cell lines and it was reported that expression of hsa-miR-320a correlated with patient prognoses. Its over- expression regulates the insulin-like growth factor-1 receptor and acts as a tumor-suppressor in glioma [49]. Lower expressions of hsa-miR-320 were observed when compared with healthy brain tissues, and also over expression of this miRNA inhibits cell proliferation and metastasis by targeting the cell cycle regulator E2F1 [50]. Most often, down regulation of mir-320 was observed and functioned as a potential biomarker for early stage detection in colorectal carcinoma [51].

While the remaining miRNAs in the top-ranked miRNA list, hsa-miR-587, hsa-miR-526a, hsa-miR-122, hsa-miR-433, and hsa-miR-574 (scored 0.53, 0.46, 0.44, 0.40 and 0.33 respectively), were not directly involved in GBM, they are, with one exception, actively associated with the major cancer types and diseases. Though, they have less experimental validations in glioblastoma, their contribution towards the survival estimation is high according to the MED analysis. Hsa-miR-526a inhibits apoptosis in tumor cells by targeting the CYLD, and plays a potential role in tumor migration and invasion via the NF-kB signaling pathway [52]. Hsa-miR-122 is frequently down-regulated in hepatocellular carcinoma, which targets peroxiredoxin 2 and induces apoptosis [53]. Hsa-miR-433 is down-regulated and is a target of tumor associated proteins GRB2 and RAB-94 in gastric cancer [54]. Hsa-miR-574 is involved in the suppression of colorectal cancer liver metastasis by negatively regulating the metastasis associated in colon cancer [55]. The lone member of the top-10 miRNA not previously associated with cancer types or diseases is hsa-miR-587. The membership on this list indicates that hsa-miR-587 may be a valuable subject of further exploration. Although these top-ranked miRNAs do not directly participate in the glioblastoma cancer, they are worthy subjects for further investigation in GBM cancer and might help in the gene target based therapies.

Besides the 10 miRNAs listed in the main effect difference results table (Table 2), several of the 14 other identified miRNAs, such as hsa-miR-223, hsa-miR-497, hsa-miR-137, hsa-miR-656 and hsa-miR-221 (scored $0.28,0.26,0.21$ and 0.20 respectively), have also been found to play a potential role in GBM progression. HsamiR-223 targets the paired box 6 (PAX6), which regulates proliferation and invasion of glioblastoma cells [56]. Hsa-miR-497 expression was associated with glioma drug resistance and it acts as a potential molecular target in glioma cells [57]. Hsa-miR-137 plays a key role in glioma, often it was downregulated. Recent investigation indicated that direct overexpression of hsa-miR-137 and delphinidin treatment effectively controlled glioblastoma growth [58]; this miRNA also induces 
apoptosis and inhibits the growth of glioma cells by targeting RAC1 [59]. Hsa-miR-656 expression levels are downregulated in glioma, and it inhibits the neurosphere formation and cell proliferation in glioma cell lines by targeting the bone morphogenetic protein -2 receptor, type-1 A (BMPR1A) [60]. Expression levels of hsa-miR221 in glioma are significantly upregulated; mir-221/222 module regulates cell proliferation and apoptosis in glioma cell lines by targeting PUMA and suppressing tumor size $[32,33]$. Hsa-miR-603 stimulates cell proliferation via $\beta$-catenin-interacting protein 1 (CTNNBIP1) and Wnt inhibitory factor 1 (WIF1) in glioma cell lines in vitro and in vivo [61].

It is the work's finding that the set of the 24 miRNA signatures can be used to estimate the survival time in patients with GBM. Additionally, the 10 top-ranked miRNAs contributed well towards survival estimation and analysis of these miRNAs revealed their functionality in various properties of cancer cell, such as proliferation, invasion and apoptosis, which can assist the understanding of mechanism of cancer progression in GBM. Several miRNAs in our study have been directly observed participating in GBM; however, a few miRNAs are not directly implicated in GBM, but they contributed towards survival estimation and many also play a key role in other major cancer types.

To measure the individual effect of these 24 identified miRNAs on survival time estimation, we used feature knock-out analysis. The 10 miRNAs, hsa-miR-222, hsamiR-345, hsa-miR-587, hsa-miR-526a, hsa-miR-335, hsamiR-122, hsa-miR-24, hsa-miR-433, hsa-miR-574, and hsa-miR-320, individually contributed correlation coefficients of $0.34,0.06,0.29,0.16,0.07,0.17,0.33,0.18,0.22$, and 0.25 respectively corresponding mean absolute error is also shown in Table 3. Correlation plots for the 10 top-ranked miRNAs are shown in Fig. 2. The remaining 14 miRNAs among the 24 are shown in Additional file 1: Figure S1.

Table 3 Individual effects of miRNAs on survival estimation

\begin{tabular}{lcc}
\hline miRNAs & Correlation coefficient & Mean absolute error (in months) \\
\hline hsa-miR-222 & 0.34 & 9.58 \\
hsa-miR-345 & 0.06 & 9.73 \\
hsa-miR-587 & 0.29 & 9.24 \\
hsa-miR-526a & 0.16 & 9.65 \\
hsa-miR-335 & 0.07 & 9.65 \\
hsa-miR-122 & 0.17 & 9.61 \\
hsa-miR-24 & 0.33 & 8.76 \\
hsa-miR-433 & 0.18 & 9.59 \\
hsa-miR-574 & 0.22 & 9.33 \\
hsa-miR-320 & 0.25 & 9.32 \\
\hline
\end{tabular}

\section{KEGG pathway}

To evaluate the biological significance of the 24 identified miRNAs involved in cancer and non-cancer pathways, we employed the KEGG pathway analysis using the DIANA tools. The 10 top-ranked miRNAs show statistical significance with cancers, such as chronic myeloid leukemia, glioma, pancreatic cancer, non-small cell lung cancer, colorectal cancer melanoma, and prostate cancer, and signaling pathways, such as Hippo signaling pathway, TGF-beta signaling pathway, thyroid hormone signaling pathway, FoxO signaling pathway, and mRNA surveillance pathway to name a few. Complete KEGG pathway analysis of these 10 miRNAs and statistical significance in different pathways and number of involved genes are shown in Table 4. The 10 top-ranked miRNAs and their target gene enrichment in cancer and signaling pathways are shown in Fig. 3 and all the 24 miRNAs gene enrichment analysis is shown in Additional file 1: Figure S2.

\section{Target gene prediction}

After identifying the miRNAs associated with survival time, we conducted target gene prediction for the set of 10 top-ranked miRNAs using miRTarBase [62]. We identified 162 non-redundant experimentally strong evidence target genes for hsa-miR-222, hsa-miR-345, hsamiR-335, hsa-miR-24, hsa-miR-433, hsa-miR-574, and hsa-miR-320 (data not shown). MiRNAs act as both tumor suppressors and oncogenes in different cancer pathways for these target genes. So, we reported the participation of each miRNA in different cancer types. Among the 10 miRNAs, seven miRNAs have experimentally validated genes and their regulation in various cancer types. Experimentally validated genes and miRNA regulation are shown in Table 5.

\section{Conclusion}

This study presents the identification of miRNA signatures with respect to their correlation with survival time in patients with GBM. Many studies used the GBM data from the TCGA data portal. However, the outcome results were accordingly not the same. In fact, the extracted miRNA profiles based on clinical follow up and filtered procedures were different across all the studies. In this work, we first developed a miRNA expression profile-based survival time estimation method called SVR-GBM, which incorporates the optimal feature selection algorithm IBCGA. SVR-GBM identified 24 miRNAs associated with the survival time in patients with GBM. Our model estimated the survival time of 247 patients with GBM and achieved a correlation coefficient of 0.76 and a mean absolute error of 0.63 years, and is comparatively better than multiple regression analysis method. In this work, 


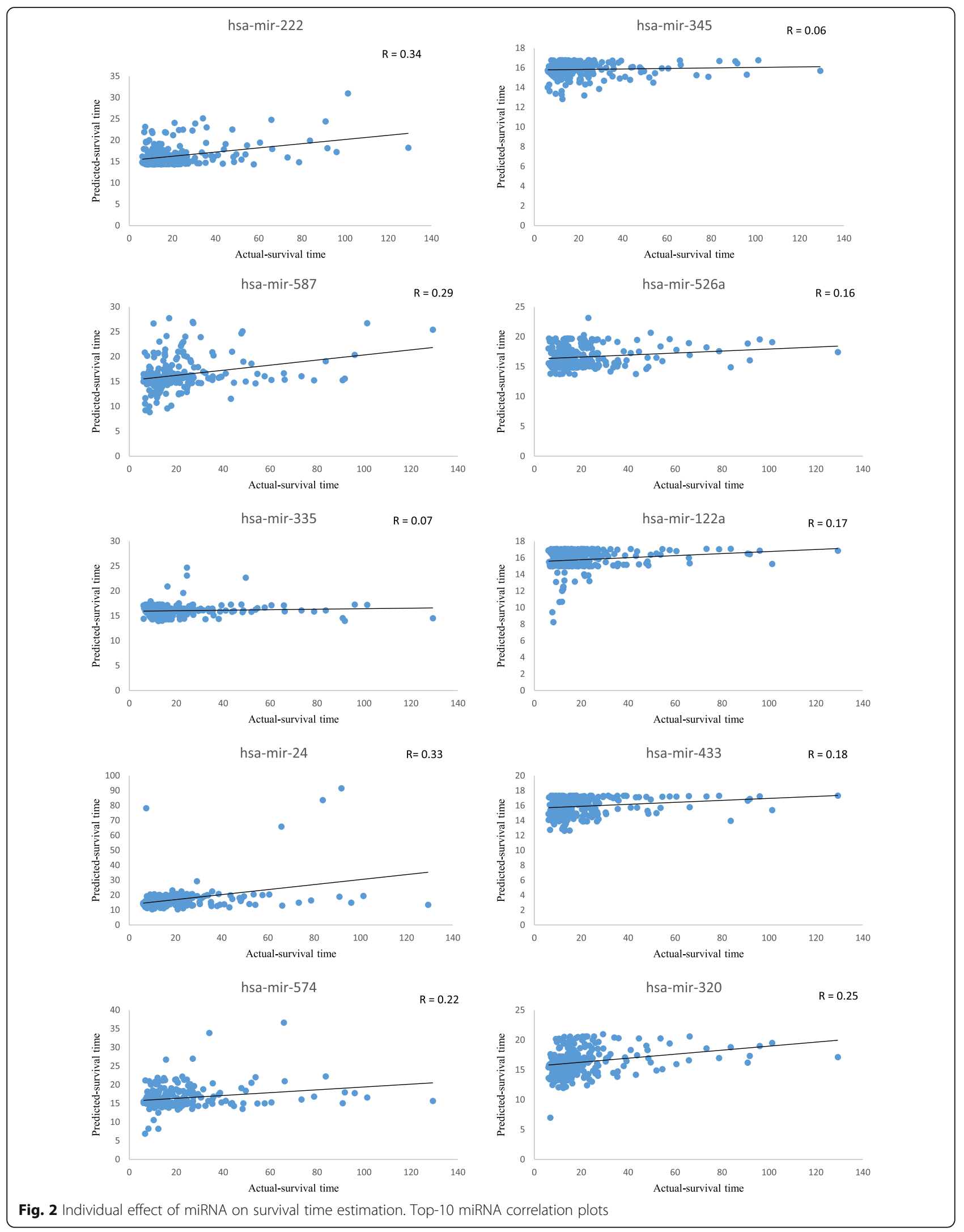


Table 4 The 10 top-ranked miRNAs and their target gene involvement in the KEGG pathway

\begin{tabular}{|c|c|c|c|}
\hline KEGG pathway & Genes & miRNAs & $p$-value \\
\hline Hippo signaling pathway & 33 & 3 & $1.72 \mathrm{E}-12$ \\
\hline Fatty acid elongation & 3 & 2 & $5.35 \mathrm{E}-12$ \\
\hline Proteoglycans in cancer & 60 & 5 & $1.36 \mathrm{E}-08$ \\
\hline Fatty acid metabolism & 2 & 2 & 1.79E-08 \\
\hline Transcriptional misregulation in cancer & 55 & 2 & $1.40 \mathrm{E}-06$ \\
\hline ECM-receptor interaction & 16 & 2 & $1.71 \mathrm{E}-06$ \\
\hline Fatty acid degradation & 1 & 1 & 3.97E-06 \\
\hline Chronic myeloid leukemia & 33 & 2 & 0.00026795 \\
\hline Glioma & 25 & 3 & 0.00035641 \\
\hline TGF-beta signaling pathway & 29 & 2 & 0.00181306 \\
\hline Adherens junction & 27 & 2 & 0.00272898 \\
\hline Biosynthesis of unsaturated fatty acids & 2 & 2 & 0.00488867 \\
\hline Viral carcinogenesis & 50 & 3 & 0.01025578 \\
\hline Pathways in cancer & 59 & 1 & 0.03687983 \\
\hline Pancreatic cancer & 26 & 2 & 0.03809962 \\
\hline Metabolism of xenobiotics by cytochrome P450 & 2 & 1 & 0.05544785 \\
\hline Signaling pathways regulating pluripotency of stem cells & 43 & 2 & 0.06508793 \\
\hline Central carbon metabolism in cancer & 24 & 2 & 0.06577197 \\
\hline Non-small cell lung cancer & 15 & 1 & 0.1204275 \\
\hline Colorectal cancer & 23 & 2 & 0.129777 \\
\hline Thyroid hormone signaling pathway & 42 & 2 & 0.1389078 \\
\hline Other types of O-glycan biosynthesis & 8 & 1 & 0.2209965 \\
\hline Lysine degradation & 4 & 1 & 0.223509 \\
\hline Spliceosome & 24 & 2 & 0.2736693 \\
\hline Small cell lung cancer & 23 & 1 & 0.2798967 \\
\hline Prostate cancer & 26 & 2 & 0.3062628 \\
\hline Melanoma & 22 & 2 & 0.3099633 \\
\hline Insulin signaling pathway & 31 & 1 & 0.3635885 \\
\hline Antigen processing and presentation & 5 & 1 & 0.4749925 \\
\hline Shigellosis & 6 & 1 & 0.5180352 \\
\hline Cell cycle & 22 & 1 & 0.5701685 \\
\hline Steroid biosynthesis & 1 & 1 & 0.6006251 \\
\hline FoxO signaling pathway & 25 & 1 & 0.6250634 \\
\hline Sulfur relay system & 2 & 2 & 0.6482195 \\
\hline Estrogen signaling pathway & 19 & 1 & 0.6860049 \\
\hline Long-term depression & 10 & 1 & 0.6946705 \\
\hline Base excision repair & 2 & 1 & 0.738435 \\
\hline Protein processing in endoplasmic reticulum & 10 & 1 & 0.7774063 \\
\hline mRNA surveillance pathway & 7 & 1 & 0.8486927 \\
\hline RNA transport & 29 & 1 & 0.8553475 \\
\hline AMPK signaling pathway & 23 & 1 & 0.859823 \\
\hline Huntington's disease & 2 & 1 & 0.9555257 \\
\hline Adipocytokine signaling pathway & 13 & 1 & 0.9654451 \\
\hline Allograft rejection & 3 & 1 & 0.9744973 \\
\hline
\end{tabular}


Table 4 The 10 top-ranked miRNAs and their target gene involvement in the KEGG pathway (Continued)

\begin{tabular}{llll}
\hline Cocaine addiction & 8 & 1 & 0.9772773 \\
Purine metabolism & 5 & 1 & 0.9896283 \\
Renin-angiotensin system & 1 & 1 & 0.9943529 \\
Valine, leucine and isoleucine degradation & 1 & 1 & 0.9945874 \\
Valine, leucine and isoleucine biosynthesis & 1 & 1 & 0.9973641 \\
\hline
\end{tabular}

miRNA expression profiles were solely used to estimate the survival time, the results were not tremendous. The model can be refined by considering other factors, such as mRNA and protein expression profiles. Furthermore, we ranked the 24 identified miRNAs based on their contribution towards the survival time estimation. The biological significance of these miRNAs was discussed, and miRNA analysis revealed their functional role in GBM cancer and other major cancer types. This study would provide a new insight into molecular therapeutic approaches to improving the therapies of GBM patients.

\section{Methods}

\section{Dataset}

All miRNA expression profiles of glioblastoma patients and corresponding clinical information were retrieved from the TCGA database. Level 3 data of 528 samples on the Agilent human 8X15k were downloaded. We followed certain criteria to retrieve samples: (i) the patients who undergone chemotherapy/radiotherapy, (ii) the patients who had survival information (days to death), (iii) the patients whose survival period equal or greater than 30 days, and (iv) elimination of duplicate entries by merging all patient lists and the corresponding survival periods. After filtering out the samples, there were a total of 247 samples with 470 miRNAs, which we used for further analysis.

\section{SVR-GBM}

We proposed a novel method SVR-GBM to predict the survival time in patients with GBM. This method also identifies the informative miRNAs to determine their functionality in GBM.

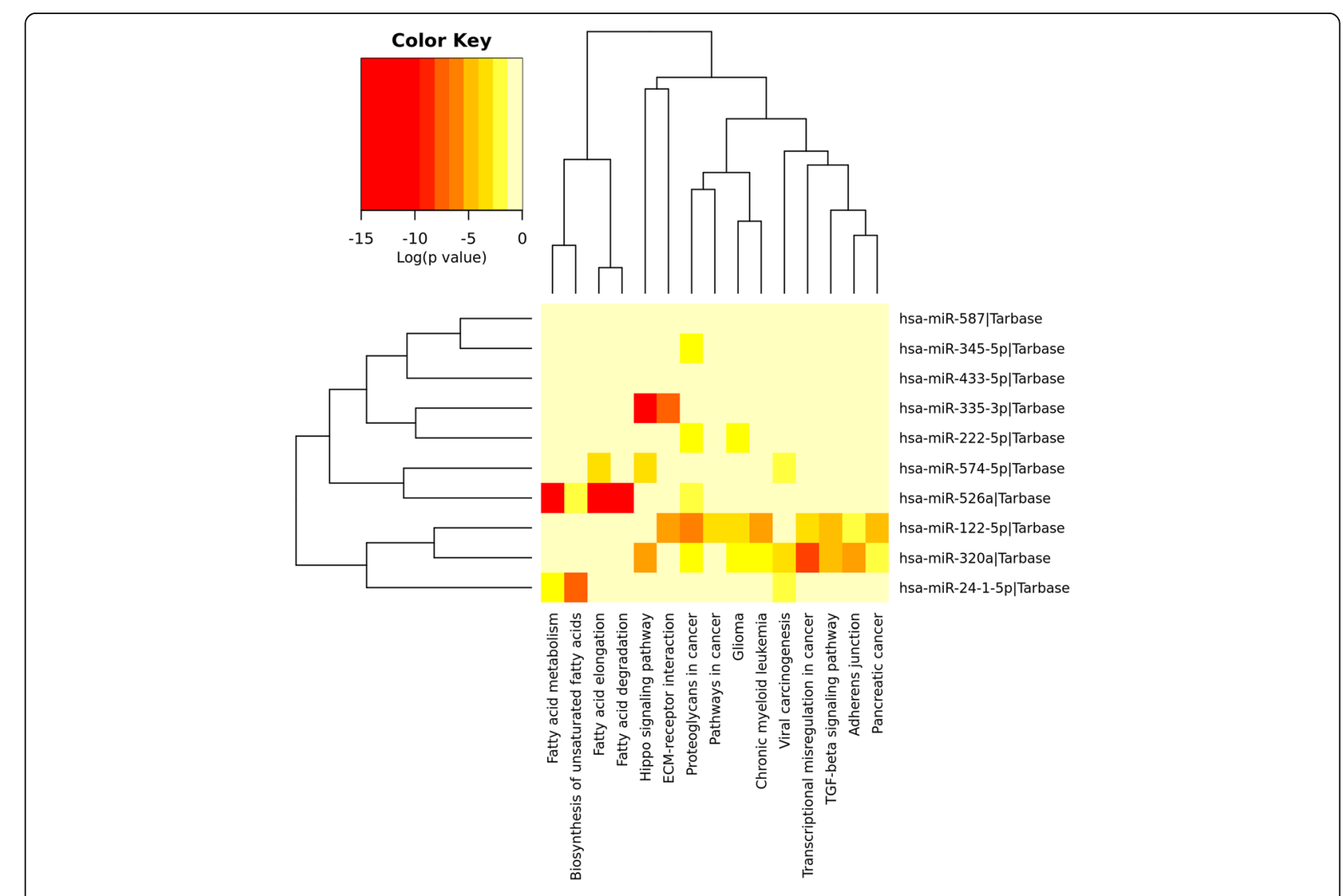

Fig. 3 Heat map of the KEGG pathway. 10 miRNA signatures involved in different cancer pathways including glioma and signaling pathways 
Table 5 Experimentally validated target genes for miRNAs

\begin{tabular}{|c|c|c|c|c|c|}
\hline miRNA & Target gene & Regulation & Validation method & Cancer & Reference \\
\hline \multirow[t]{7}{*}{ hsa-miR-222 } & GJA1 & down & Immunofluorescence, Western Blot, Luciferase Reporter Assay & Glioblastoma & {$[35]$} \\
\hline & CDKN1C & down & Luciferase Reporter Assay & Glioblastoma & [69] \\
\hline & P27kip1 & down & Western Blot & Glioblastoma & [32] \\
\hline & DICER1 & down & Luciferase Reporter Assay & Breast cancer & [70] \\
\hline & TIMP3 & Down & Luciferase Reporter Assay, qPCR, Western Blot & Lung cancer & [36] \\
\hline & CDKN1B & Down & Luciferase Reporter Assay & Thyroid carcinoma & [71] \\
\hline & PPP2R2A & Down & Luciferase Reporter Assay & Lung cancer & [72] \\
\hline \multirow[t]{3}{*}{ hsa-miR-345 } & MCL-1 and BCL2L2 & Up & microarray & Lung cancer & [73] \\
\hline & Smad1 & Down & microarray & Prostate cancer & [39] \\
\hline & BAG3 & Down & Luciferase reporter assay and western blot & colorectal cancer & {$[40]$} \\
\hline \multirow[t]{2}{*}{ hsa-miR-335 } & SOX4 & Down & Northern blot, qRT-PCR etc. & breast cancer & [74] \\
\hline & TGF- $\beta$ & Down & qRT-PCR & Neuroblastoma & [44] \\
\hline hsa-miR-122a & CCNG1 & Down & Northern blot, qRT-PCR & hepatocellular carcinoma & [75] \\
\hline \multirow[t]{2}{*}{ hsa-miR-24 } & ST7L & Up & qRT-PCR & glioma & [45] \\
\hline & MXI1 & Up & MTT assay & glioma & {$[46]$} \\
\hline hsa-miR-433 & FGF20 & - & - Northern blot, qRT-PCR etc. & Parkinson's disease & {$[76]$} \\
\hline \multirow[t]{2}{*}{ hsa-miR-320 } & TfR-1 & Down & northern blot, qRT-PCR & acute myeloid leukemia & [77] \\
\hline & $\mathrm{MCl}-1, \mathrm{BCL} 2$ & Down & northern blot, qRT-PCR & cholangiocarcinoma & [78] \\
\hline
\end{tabular}

\section{Integration of IBCGA and SVR for miRNA selection and modeling}

Support vector machine (SVM) is based on statistical learning theory and successfully applied to classification and regression problems [63]. In this work, we approached support vector regression method to estimate the survival time in patients with GBM. The $v$ support vector regression (SVR) presents the good performance because it relies on the number of support vectors and training error. Given a set of data points, $\left(x_{1}, y_{1}\right),\left(x_{2}, y 2\right) \ldots\left(x_{m}, y_{m}\right)$, where $x_{i} \in R^{n}$ is an input and $y_{i} \in R^{1}$ is a target output. The optimization problem of the $v$-SVR can be defined as follows.

$$
\min \left[\left\{\frac{1}{2} w^{T}\left(\phi\left(x_{i}\right)+b\right)+C\left(v \varepsilon+\frac{1}{m}\left(\sum_{i=1}^{m}\left(\xi_{i}+\xi_{i}^{*}\right)\right)\right)\right\}\right]
$$

where $\xi_{i} \geq 0, \quad \xi_{i}^{*} \geq 0, \varepsilon \geq 0 ; \mathrm{i}=1,2, \ldots, \mathrm{m} ; \quad$ and $\mathrm{b}$ is a constant.

Here, $0 \leq v \leq 1$, and $C$ is the regularization parameter. The $\varepsilon$-insensitive loss function means that if $w^{T} \varnothing\left(x_{i}\right)$ is in the range of $\mathrm{y} \pm \varepsilon$ no loss is considered. The $\mathrm{y} \pm \varepsilon$ is as known as the soft margin where $v$ is an upper bound on the fraction of margin errors and a lower bound of the fraction of support vectors . In this work, the LibSVM package was used for implementation ofv-SVR [64]. To select a minimal set of informative features from a large number of candidate features problem, the inheritable bi-objective combinatorial genetic algorithm (IBCGA) [28] was used. In this work, we incorporated the optimal feature selection algorithm IBCGA and $v$-SVR to obtain an optimized model. The parameters for designing the SVR model to be optimised simultaneously by the IBCGA are the $n$ binary variables for selecting informative miRNAs and tuning parameters $C, \gamma$ and $v$ of the SVR. The chromosome of the IBCGA comprises $n$ binary genes $f_{\mathrm{i}}$ to select $m$ miRNA and three 4-bit genes for encoding $\gamma, \mathrm{C}$, and $v$ of the SVR. The i-th miRNA is excluded from the SVR regression model if $f_{i}=0$ and included if $f_{i}=1$. The sum of $f_{i}$ is equal to $m$. The IBCGA can simultaneously obtain a set of solutions, $X_{p}$ where $r=r_{\text {start }}, r_{\text {start }}+1, \ldots, r_{\text {end }}$ in a single run. In this work, the parameter values are $r_{\text {start }}=10, r_{\text {end }}=50$, $N_{\text {pop }}=50, P_{c}=0.8, P_{m}=0.05$, and Gmax $=60$ [28].

To maximize the estimation accuracy in terms of Pearson's correlation coefficient $(r)$ used as the fitness function, we employed 10-fold cross validation (10-CV) to measure the performance of SVR-GBM in terms of Pearson's correlation coefficient and mean absolute error between the predicted survival time and real survival time.

The Pearson's correlation coefficient $(r)$ can be formulated as follows

$$
r=\frac{\sum_{i=1}^{N}\left(x_{i}-\bar{x}\right)\left(y_{i}-\bar{y}\right)}{\sqrt{\left\lfloor\sum_{i=1}^{N}\left(x_{i}-\bar{x}\right)^{2}\right\rfloor\left[\sum_{i=1}^{N}\left(y_{i}-\bar{y}\right)^{2}\right]}}
$$


where $x_{\mathrm{i}}$ and $y_{\mathrm{i}}$ are real and predicted survival time of the $i^{\text {th }}$ miRNA, and $\bar{x}$ and $\bar{y}$ are their corresponding means. Here $N$ is the total number of miRNAs in the sample.

The mean absolute error (MAE) is described by

$$
M A E=\frac{1}{N} \sum_{i=1}^{N}\left|y_{i}-x_{i}\right|^{2}
$$

The standard error of estimates $(S E E)$ is defined as

$$
S E E=\sqrt{\frac{\sum\left(y_{i}-x_{i}\right)^{2}}{n-2}}
$$

where $y_{\mathrm{i}}$ is estimated value and $x_{\mathrm{i}}$ is actual value, $n$ is number of observations.

The customised IBCGA is described below.

Step 1) (Initialization) Generate an initial population of $N_{\text {pop }}$ individuals randomly. All the $n$ binary genes $f_{\mathrm{i}}$ have $r$ 1's and $n-r$ 0's, where $r=r_{\text {start }}$.

Step 2) (Evaluation) Evaluate all individuals using the fitness function.

Step 3) (Selection) Use a tournament selection method that selects the winner from two randomly selected individuals to form a mating pool.

Step 4) (Crossover) Select $P_{c} \cdot N_{\text {pop }}$ parents from the mating pool to perform the orthogonal array crossover, where $P_{c}$ is the crossover probability.

Step 5) (Mutation) The traditional mutation operator is applied to the randomly selected $P_{m} \cdot N_{p o p}$ individuals except the best individual, where $P_{m}$ is the mutation probability.

Step 6) (Termination test) If the stopping condition of performing $G_{\text {max }}$ generations for obtaining the solution $X_{r}$ is satisfied, output the best individual as $X_{r}$ Otherwise, go to Step 2.

Step 7) (Inheritance) If $r<r_{\text {end }}$, randomly change one bit in the binary genes $f_{i}$ for each individual from 0 to 1 ; increase the number $r$ by one, and go to Step 2 . Otherwise, stop the algorithm.

Step 8) (Output) Let $m$ equal the value of $r$ having the best fitness value. Output the $m$ miRNAs and the corresponding SVR model.

\section{Multiple linear regression}

We employed a multiple regression technique to estimate the survival time. Stepwise feature addition procedure has been used for feature selection. In multiple linear regression, every value of the independent variable $x$ is associated with the dependent variable value $y$ [65]. A general multiple linear regression can be defined as

$$
y_{i}=\beta_{0}+\beta_{1} x_{1}+\beta_{2} x_{2}+\cdots+\beta_{n} x_{n}+\varepsilon .
$$

where $y_{i}$ is the dependent variable; $x_{1}, x_{2}, \ldots, x_{\mathrm{n}}$ are the independent variables; $\beta_{0}, \beta_{1}, \beta_{2}, \ldots, \beta_{n}$ are the regression coefficients; $n$ denotes the number of terms in the model, and $\varepsilon$ is the error term.

\section{Diana tools}

We employed miRNA pathway analysis using DIANAmirpath webserver [66] which utilized DIANA-Tarbase algorithm to predict the miRNA target. In order to estimate the specificity of results, we performed the pathway analysis for all identified miRNAs. In the mirpath tool, we selected the pathway union feature to identify the specific targeted KEGG pathway for each identified miRNA. The mirpath server employs enrichment analysis and measures the significance levels ( $p$-value) between identified miRNAs and corresponding pathways using Fisher's exact test. The results of this analysis indicate that the probability of particular pathway is notably enriched with targeted by at least one selected miRNA.

\section{Gene Target prediction}

We used miRTarBase [62] and Tarbase [67] to predict the experimentally validated gene targets. Mir2 disease [68] was used to identify the cancer related miRNAs.

\section{Additional file}

Additional file 1: Additional file contains the following Figures and Tables. Figure S1. Individual effect of miRNA on survival time estimation. Correlation plots for 14 miRNAs Figure S2. Heat map of the KEGG pathway. Identified 24miRNA signatures involved in different cancer pathway and signaling pathways. Table S1. 24 miRNAs and their gene enrichment in the KEGG pathway. (PDF $360 \mathrm{~kb}$ )

\section{Acknowledgements}

This work was funded by National Science Council of Taiwan under the contract numbers MOST-105-2221-E- 009-138-MY2 and 105-2627-M-009-008-, and "Center for Bioinformatics Research of Aiming for the Top University Program" of the National Chiao Tung University and Ministry of Education, Taiwan, R.O.C. for the project 105W962. The funders had no role in study design, data collection and analysis, decision to publish, or preparation of the manuscript.

\section{Declarations}

This article has been published as part of BMC Genomics Volume 17 Supplement 13, 2016: 15th International Conference On Bioinformatics (INCOB 2016). The full contents of the supplement are available online at https://bmcgenet.biomedcentral.com/articles/supplements/volume-17supplement-13.

\section{Funding}

Publication charges for this article have been funded by the National Science Council of Taiwan, the National Chiao Tung University and the Ministry of Education, Taiwan, R.O.C.

\section{Availability of data and materials}

All the data used in this analysis can be found at TCGA data portal. [https://cancergenome.nih.gov/].

\section{Authors' contributions}

Yerukala Sathipati Srinivasulu (YSS) and Shinn-Ying Ho (SYH) designed the system, participated in manuscript preparation, and carried out the detail study. Hui-Ling Huang $(H L H)$ participated in the design of the system, 
implemented programs, and discussed the results. All authors have read and approved the final manuscript.

\section{Consent for publication}

Not applicable.

\section{Competing interests}

The authors declare that they have no competing interests.

\section{Ethics approval and consent to participate}

Not applicable.

\section{Published: 22 December 2016}

\section{References}

1. Diehn M, Nardini C, Wang DS, McGovern S, Jayaraman M, Liang $Y$, Aldape K, Cha S, Kuo MD. Identification of noninvasive imaging surrogates for brain tumor gene-expression modules. Proc Natl Acad Sci U S A. 2008;105(13):5213-8.

2. Kleihues $P$, Ohgaki $H$. Phenotype vs genotype in the evolution of astrocytic brain tumors. Toxicol Pathol. 2000;28(1):164-70.

3. Sathornsumetee S, Rich JN. Designer therapies for glioblastoma multiforme. Ann N Y Acad Sci. 2008;1142:108-32.

4. Stupp R, Hegi ME, Mason WP, van den Bent MJ, Taphoorn MJB, Janzer RC, Ludwin SK, Allgeier A, Fisher B, Belanger K, et al. Effects of radiotherapy with concomitant and adjuvant temozolomide versus radiotherapy alone on survival in glioblastoma in a randomised phase III study: 5-year analysis of the EORTC-NCIC trial. Lancet Oncol. 2009;10(5):459-66.

5. Bartel DP. MicroRNAs: genomics, biogenesis, mechanism, and function. Cell. 2004;116(2):281-97.

6. Ambros $V$. The functions of animal microRNAs. Nature. 2004; 431(7006):350-5.

7. Chen CZ, Li L, Lodish HF, Bartel DP. MicroRNAs modulate hematopoietic lineage differentiation. Science. 2004;303(5654):83-6.

8. Brennecke J, Hipfner DR, Stark A, Russell RB, Cohen SM. Bantam encodes a developmentally regulated microRNA that controls cell proliferation and regulates the proapoptotic gene hid in Drosophila. Cell. 2003;113(1):25-36.

9. Poy MN, Eliasson L, Krutzfeldt J, Kuwajima S, Ma X, Macdonald PE, Pfeffer S, Tuschl T, Rajewsky N, Rorsman P, et al. A pancreatic islet-specific microRNA regulates insulin secretion. Nature. 2004:432(7014):226-30.

10. Calin GA, Dumitru CD, Shimizu M, Bichi R, Zupo S, Noch E, Aldler H, Rattan $\mathrm{S}$, Keating M, Rai $\mathrm{K}$, et al. Frequent deletions and down-regulation of microRNA genes miR15 and miR16 at 13q14 in chronic lymphocytic leukemia. Proc Natl Acad Sci U S A. 2002;99(24):15524-9.

11. Hermansen SK, Kristensen BW. MicroRNA biomarkers in glioblastoma. J Neurooncol. 2013:114(1):13-23.

12. Michael MZ, SM OC, van Holst Pellekaan NG, Young GP, James RJ. Reduced accumulation of specific microRNAs in colorectal neoplasia. Mol Cancer Res. 2003;1(12):882-91.

13. Takamizawa J, Konishi H, Yanagisawa K, Tomida S, Osada H, Endoh $H_{\text {, }}$ Harano T, Yatabe $Y$, Nagino M, Nimura Y, et al. Reduced expression of the let-7 microRNAs in human lung cancers in association with shortened postoperative survival. Cancer Res. 2004;64(11):3753-6.

14. Mischel PS, Shai R, Shi T, Horvath S, Lu KV, Choe G, Seligson D, Kremen TJ, Palotie A, Liau LM, et al. Identification of molecular subtypes of glioblastoma by gene expression profiling. Oncogene. 2003;22(15):2361-73.

15. Rickman DS, Bobek MP, Misek DE, Kuick R, Blaivas M, Kurnit DM, Taylor J, Hanash SM. Distinctive molecular profiles of high-grade and low-grade gliomas based on oligonucleotide microarray analysis. Cancer Res. 2001; 61(18):6885-91.

16. Shai R, Shi T, Kremen TJ, Horvath S, Liau LM, Cloughesy TF, Mischel PS, Nelson SF. Gene expression profiling identifies molecular subtypes of gliomas. Oncogene. 2003;22(31):4918-23.

17. Kim S, Dougherty ER, Shmulevich I, Hess KR, Hamilton SR, Trent JM, Fuller GN, Zhang W. Identification of combination gene sets for glioma classification. Mol Cancer Ther. 2002;1(13):1229-36.

18. Fuller GN, Mircean C, Tabus I, Taylor E, Sawaya R, Bruner JM, Shmulevich I, Zhang W. Molecular voting for glioma classification reflecting heterogeneity in the continuum of cancer progression. Oncol Rep. 2005;14(3):651-6.
19. Lu J, Getz G, Miska EA, Alvarez-Saavedra E, Lamb J, Peck D, Sweet-Cordero A, Ebert BL, Mak RH, Ferrando AA, et al. MicroRNA expression profiles classify human cancers. Nature. 2005;435(7043):834-8.

20. Guan Y, Mizoguchi M, Yoshimoto K, Hata N, Shono T, Suzuki SO, Araki Y, Kuga D, Nakamizo A, Amano T, et al. MiRNA-196 is upregulated in glioblastoma but not in anaplastic astrocytoma and has prognostic significance. Clin Cancer Res. 2010;16(16):4289-97.

21. Kim H, Huang W, Jiang X, Pennicooke B, Park PJ, Johnson MD. Integrative genome analysis reveals an oncomir/oncogene cluster regulating glioblastoma survivorship. Proc Natl Acad Sci U S A. 2010;107(5):2183-8.

22. Ma R, Yan W, Zhang G, Lv H, Liu Z, Fang F, Zhang W, Zhang J, Tao T, You Y, et al. Upregulation of miR-196b Confers a Poor Prognosis in Glioblastoma Patients via Inducing a Proliferative Phenotype. PLoS One. 2012;7(6):e38096.

23. Gwak HS, Kim TH, Jo GH, Kim YJ, Kwak HJ, Kim JH, Yin J, Yoo H, Lee SH, Park JB. Silencing of microRNA-21 confers radio-sensitivity through inhibition of the PI3K AKT pathway and enhancing autophagy in malignant glioma cell lines. PLoS One. 2012;7(10):e47449.

24. Zhang S, Wan Y, Pan T, Gu X, Qian C, Sun G, Sun L, Xiang Y, Wang Z, Shi L. MicroRNA-21 inhibitor sensitizes human glioblastoma U251 stem cells to chemotherapeutic drug temozolomide. J Mol Neurosci. 2012; 47(2):346-56

25. Teplyuk NM, Mollenhauer B, Gabriely G, Giese A, Kim E, Smolsky M, Kim RY, Saria MG, Pastorino S, Kesari S, et al. MicroRNAs in cerebrospinal fluid identify glioblastoma and metastatic brain cancers and reflect disease activity. Neuro Oncol. 2012;14(6):689-700.

26. Roth P, Wischhusen J, Happold C, Chandran PA, Hofer S, Eisele G, Weller M, Keller A. A specific miRNA signature in the peripheral blood of glioblastoma patients. J Neurochem. 2011;118(3):449-57.

27. Nutt CL, Mani DR, Betensky RA, Tamayo P, Cairncross JG, Ladd C, Pohl U, Hartmann C, McLaughlin ME, Batchelor TT, et al. Gene expression-based classification of malignant gliomas correlates better with survival than histological classification. Cancer Res. 2003;63(7):1602-7.

28. Ho SY, Chen JH, Huang MH. Inheritable genetic algorithm for biobjective $0 /$ 1 combinatorial optimization problems and its applications. IEEE Trans Syst Man Cybern B Cybern. 2004;34:609-20.

29. Hocking RR. A Biometrics Invited Paper. The Analysis and Selection of Variables in Linear Regression. Biometrics. 1976;32(1):1-49.

30. Zou H, Hastie T. Regularization and variable selection via the elastic net. J R Stat Soc Ser B (Stat Methodol). 2005;67(2):301-20.

31. Tung C-W, Ho S-Y. Computational identification of ubiquitylation sites from protein sequences. BMC Bioinf. 2008;9(1):1-15.

32. Zhang C, Kang C, You Y, Pu P, Yang W, Zhao P, Wang G, Zhang A, Jia Z, Han L, et al. Co-suppression of miR-221/222 cluster suppresses human glioma cell growth by targeting p27kip1 in vitro and in vivo. Int J Oncol. 2009;34(6):1653-60.

33. Zhang C-Z, Zhang J-X, Zhang A-L, Shi Z-D, Han L, Jia Z-F, Yang W-D, Wang $\mathrm{G}-\mathrm{X}$, Jiang T, You Y-P, et al. MiR-221 and miR-222 target PUMA to induce cell survival in glioblastoma. Mol Cancer. 2010;9(1):1-9.

34. Yang F, Wang W, Zhou C, Xi W, Yuan L, Chen X, Li Y, Yang A, Zhang J, Wang T. MiR-221/222 promote human glioma cell invasion and angiogenesis by targeting TIMP2. Tumour Biol. 2015;36(5):3763-73.

35. Hao J, Zhang C, Zhang A, Wang K, Jia Z, Wang G, Han L, Kang C, Pu P. miR-221/222 is the regulator of $C \times 43$ expression in human glioblastoma cells. Oncol Rep. 2012;27(5):1504-10.

36. Garofalo M, Di Leva G, Romano G, Nuovo G, Suh SS, Ngankeu A, Taccioli C, Pichiorri $F$, Alder $H$, Secchiero $P$, et al. miR-221\&222 regulate TRAll resistance and enhance tumorigenicity through PTEN and TIMP3 downregulation. Cancer Cell. 2009;16(6):498-509.

37. Zinn PO, Sathyan P, Mahajan B, Bruyere J, Hegi M, Majumder S, Colen RR. A Novel Volume-Age-KPS (VAK) Glioblastoma Classification Identifies a Prognostic Cognate microRNA-Gene Signature. PLoS One. 2012;7(8):e41522.

38. Chen L, Li X, Chen X. Prognostic significance of tissue miR-345 downregulation in non-small cell lung cancer. Int J Clin Exp Med. 2015;8(11):20971-6.

39. Q-g C, Zhou W, Han T, Du S-q, Li Z-h, Zhang Z, Shan G-y, Kong C-z. MiR-345 suppresses proliferation, migration and invasion by targeting Smad1 in human prostate cancer. J Cancer Res Clin Oncol. 2016;142(1):213-24.

40. Tang JT, Wang JL, Du W, Hong J, Zhao SL, Wang YC, Xiong H, Chen HM, Fang JY. MicroRNA 345, a methylation-sensitive microRNA is involved in cell proliferation and invasion in human colorectal cancer. Carcinogenesis. 2011;32(8):1207-15. 
41. Jiang J, Sun X, Wang W, Jin X, Bo X, Li Z, Bian A, Jiu J, Wang X, Liu D, et al Tumor microRNA-335 expression is associated with poor prognosis in human glioma. Med Oncol. 2012;29(5):3472-7.

42. Gao Y, Zeng F, Wu JY, Li HY, Fan JJ, Mai L, Zhang J, Ma DM, Li Y, Song FZ. MiR-335 inhibits migration of breast cancer cells through targeting oncoprotein c-Met. Tumour Biol. 2015;36(4):2875-83.

43. Cao J, Cai J, Huang D, Han Q, Yang Q, Li T, Ding H, Wang Z. miR-335 represents an invasion suppressor gene in ovarian cancer by targeting $\mathrm{BCl}$ w. Oncol Rep. 2013;30(2):701-6.

44. Lynch J, Fay J, Meehan M, Bryan K, Watters KM, Murphy DM, Stallings RL. MiRNA-335 suppresses neuroblastoma cell invasiveness by direct targeting of multiple genes from the non-canonical TGF-beta signalling pathway. Carcinogenesis. 2012;33(5):976-85.

45. Chen L, Zhang A, Li Y, Zhang K, Han L, Du W, Yan W, Li R, Wang Y, Wang K, et al. MiR-24 regulates the proliferation and invasion of glioma by ST7L via beta-catenin/Tcf-4 signaling. Cancer Lett. 2013;329(2):174-80.

46. Xu W, Liu M, Peng X, Zhou P, Zhou J, Xu K, Xu H, Jiang S. miR-24-3p and miR-27a-3p promote cell proliferation in glioma cells via cooperative regulation of MXI1. Int J Oncol. 2013;42(2):757-66.

47. Lu K, Wang J, Song Y, Zhao S, Liu H, Tang D, Pan B, Zhao H, Zhang Q. miRNA-24$3 p$ promotes cell proliferation and inhibits apoptosis in human breast cancer by targeting p27Kip1. Oncol Rep. 2015;34(2):995-1002.

48. Zhao G, Liu L, Zhao T, Jin S, Jiang S, Cao S, Han J, Xin Y, Dong Q, Liu X, et al. Upregulation of miR-24 promotes cell proliferation by targeting NAIF1 in non-small cell lung cancer. Tumour Biol. 2015;36(5):3693-701.

49. Guo T, Feng Y, Liu Q, Yang X, Jiang T, Chen Y, Zhang Q. MicroRNA-320a suppresses in GBM patients and modulates glioma cell functions by targeting IGF-1R. Tumour Biol. 2014;35(11):11269-75.

50. Sun JY, Xiao WZ, Wang F, Wang YQ, Zhu YH, Wu YF, Miao ZL, Lin YC. MicroRNA-320 inhibits cell proliferation in glioma by targeting E2F1. Mol Med Rep. 2015;12(2):2355-9.

51. Fang $Z$, Tang J, Bai $Y$, Lin $H$, You $H$, Jin $H$, Lin L, You $P$, Li J, Dai $Z$, et al. Plasma levels of microRNA-24, microRNA-320a, and microRNA-423$5 p$ are potential biomarkers for colorectal carcinoma. J Exp Clin Cancer Res. 2015;34(1):1-10.

52. Yang X, Wang C, Xu C, Yan Z, Wei C, Guan K, Ma S, Cao Y, Liu L, Zou D, et al. miR-526a regulates apoptotic cell growth in human carcinoma cells. Mol Cell Biochem. 2015:407(1-2):69-76.

53. Diao S, Zhang JF, Wang H, He ML, Lin MC, Chen Y, Kung HF. Proteomic identification of microRNA-122a target proteins in hepatocellular carcinoma. Proteomics. 2010;10(20):3723-31.

54. Luo H, Zhang H, Zhang Z, Zhang X, Ning B, Guo J, Nie N, Liu B, Wu X Down-regulated miR-9 and miR-433 in human gastric carcinoma. J Exp Clin Cancer Res. 2009;28:82.

55. Cui Z, Tang J, Chen J, Wang Z. Hsa-miR-574-5p negatively regulates MACC-1 expression to suppress colorectal cancer liver metastasis. Cancer Cell Int 2014;14(1):1-9.

56. Huang BS, Luo QZ, Han Y, Li XB, Cao LJ, Wu LX. microRNA-223 promotes the growth and invasion of glioblastoma cells by targeting tumor suppressor PAX6. Oncol Rep. 2013;30(5):2263-9.

57. Lan J, Xue Y, Chen H, Zhao S, Wu Z, Fang J, Han C, Lou M. Hypoxia-induced miR-497 decreases glioma cell sensitivity to TMZ by inhibiting apoptosis. FEBS Lett. 2014;588(18):3333-9.

58. Chakrabarti M, Ray SK. Direct transfection of miR-137 mimics is more effective than DNA demethylation of miR-137 promoter to augment anti-tumor mechanisms of delphinidin in human glioblastoma U87MG and LN18 cells. Gene. 2015;573(1):141-52.

59. Sun G, Cao Y, Shi L, Sun L, Wang Y, Chen C, Wan Z, Fu L, You Y. Overexpressed miRNA-137 Inhibits Human Glioma Cells Growth by Targeting Rac1. Cancer Biother Radiopharm. 2013;28(4):327-34.

60. Guo M, Jiang Z, Zhang X, Lu D, Ha AD, Sun J, Du W, Wu Z, Hu L, Khadarian $K$, et al. miR-656 inhibits glioma tumorigenesis through repression of BMPR1A. Carcinogenesis. 2014;35(8):1698-706.

61. Guo M, Zhang X, Wang G, Sun J, Jiang Z, Khadarian K, Yu S, Zhao Y, Xie C, Zhang $K$, et al. miR-603 promotes glioma cell growth via Wnt/B-catenin pathway by inhibiting WIF1 and CTNNBIP1. Cancer Lett. 2015;360(1):76-86

62. Hsu SD, Tseng YT, Shrestha S, Lin YL, Khaleel A, Chou CH, Chu CF, Huang HY, Lin CM, Ho SY, et al. miRTarBase update: an information resource for experimentally validated miRNA-target interactions. Nucleic Acids Res. 2014; 42(Database issue):D78-85.
63. Vapnik VN. An overview of statistical learning theory. IEEE Trans Neural Netw. 1999;10(5):988-99.

64. Chang C-C, Lin C-J. LIBSVM: A library for support vector machines. ACM Trans Intell Syst Technol. 2011;2(3):1-27.

65. Aiken LS, West SG, Pitts SC. Multiple Linear Regression. Handbook of Psychology. 2003;Four:19:481-507.

66. Paraskevopoulou MD, Georgakilas G, Kostoulas N, Vlachos IS, Vergoulis T, Reczko M, Filippidis C, Dalamagas T, Hatzigeorgiou AG. DIANA-microT web server v5.0: service integration into miRNA functional analysis workflows. Nucleic Acids Res. 2013;41(Web Server issue):W169-73.

67. Vlachos IS, Paraskevopoulou MD, Karagkouni D, Georgakilas G, Vergoulis T, Kanellos I, Anastasopoulos IL, Maniou S, Karathanou K, Kalfakakou D, et al. DIANA-TarBase v7.0: indexing more than half a million experimentally supported miRNA:mRNA interactions. Nucleic Acids Res. 2015:43(Database issue):D153-9.

68. Jiang Q, Wang Y, Hao Y, Juan L, Teng M, Zhang X, Li M, Wang G, Liu Y. miR2Disease: a manually curated database for microRNA deregulation in human disease. Nucleic Acids Res. 2009;37(Database issue):D98-D104.

69. Medina R, Zaidi SK, Liu CG, Stein JL, van Wijnen AJ, Croce CM, Stein GS. MicroRNAs 221 and 222 bypass quiescence and compromise cell survival. Cancer Res. 2008;68(8):2773-80.

70. Cochrane DR, Cittelly DM, Howe EN, Spoelstra NS, McKinsey EL, LaPara K, Elias A, Yee D, Richer JK. MicroRNAs link estrogen receptor alpha status and Dicer levels in breast cancer. Horm Cancer. 2010;1(6):306-19.

71. Visone R, Russo L, Pallante P, De Martino I, Ferraro A, Leone V, Borbone E, Petrocca F, Alder H, Croce CM, et al. MicroRNAs (miR)-221 and miR-222, both overexpressed in human thyroid papillary carcinomas, regulate p27Kip1 protein levels and cell cycle. Endocr Relat Cancer. 2007;14(3):791-8.

72. Zhang Y, Ma T, Yang S, Xia M, Xu J, An H, Yang Y, Li S. High-mobility group A1 proteins enhance the expression of the oncogenic miR-222 in lung cancer cells. Mol Cell Biochem. 2011;357(1-2):363-71.

73. Crawford M, Batte K, Yu L, Wu X, Nuovo GJ, Marsh CB, Otterson GA, Nana-Sinkam SP. MicroRNA 133B targets pro-survival molecules MCL-1 and BCL2L2 in lung cancer. Biochem Biophys Res Commun. 2009;388(3):483-9.

74. Tavazoie SF, Alarcon C, Oskarsson T, Padua D, Wang Q, Bos PD, Gerald WL, Massague J. Endogenous human microRNAs that suppress breast cancer metastasis. Nature. 2008:451(7175):147-52.

75. Gramantieri L, Ferracin M, Fornari F, Veronese A, Sabbioni S, Liu CG, Calin GA, Giovannini C, Ferrazzi E, Grazi GL, et al. Cyclin G1 is a target of miR122a, a microRNA frequently down-regulated in human hepatocellular carcinoma. Cancer Res. 2007;67(13):6092-9.

76. Wang G, van der Walt JM, Mayhew G, Li YJ, Zuchner S, Scott WK, Martin ER, Vance JM. Variation in the miRNA-433 binding site of FGF20 confers risk for Parkinson disease by overexpression of alpha-synuclein. Am J Hum Genet. 2008;82(2):283-9.

77. Schaar DG, Medina DJ, Moore DF, Strair RK, Ting Y. miR-320 targets transferrin receptor 1 (CD71) and inhibits cell proliferation. Exp Hematol. 2009:37(2):245-55

78. Chen L, Yan HX, Yang W, Hu L, Yu LX, Liu Q, Li L, Huang DD, Ding J, Shen F, et al. The role of microRNA expression pattern in human intrahepatic cholangiocarcinoma. J Hepatol. 2009;50(2):358-69.

\section{Submit your next manuscript to BioMed Central and we will help you at every step:}

- We accept pre-submission inquiries

- Our selector tool helps you to find the most relevant journal

- We provide round the clock customer support

- Convenient online submission

- Thorough peer review

- Inclusion in PubMed and all major indexing services

- Maximum visibility for your research

Submit your manuscript at www.biomedcentral.com/submit 